\title{
Correlation of grade of urothelial cell carcinomas and DNA histogram features assessed by flow cytometry and automated image cytometry
}

\author{
Marco G.W. Bol a , Jan P.A. Baak ${ }^{\text {a,b,* }}$, Bianca v. Diermen ${ }^{\text {a }}$, E.A.M. Janssen ${ }^{\text {a }}$, \\ Susanne B.K. Buhr-Wildhagen ${ }^{\text {a }}$ and Kjell-Henning Kjellevold ${ }^{\text {a }}$ \\ ${ }^{a}$ Department of Pathology, SIR Hospital, Stavanger, Norway \\ ${ }^{\mathrm{b}}$ Free University, Amsterdam, The Netherlands
}

Received 10 June 2002

Accepted 3 March 2003

\begin{abstract}
Objective: To analyse how DNA ploidy and S-phase fraction (SPF) by flow cytometry (FCM) and an optimised fully automatic DNA image cytometer (ICM) correlate with grade in TaT1 urothelial cell carcinomas (UC) of the urinary bladder. Materials and methods: Two-hundred-and twenty-eight consensus cases were analysed. Single cell suspensions were stained (DAPI for FCM, Feulgen for ICM). There was enough material for both FCM and ICM in 202 of these cases. FCM and optimised ICM measurements were performed on the 202 UCs. To discriminate between different grades, single- and multivariate analyses was performed on DNA histogram features obtained with the MultiCycle program (using DNA index (DI) and SPF). Results: Overall measurement time of the adapted ICM method was 10.7 minutes per case (range 5.9-29.8 min.) and required little additional interactive object rejection (average 152 objects (84-298) on 3000 objects per case measured, which took 9.9 minutes on average, range 8.3-15.5 minutes). The ICM histograms looked much "cleaner" with less noise than the FCM graphs. The coefficient of variation (CV) of the diploid peak for ICM (5.4\%) was significantly lower than for FCM (5.9\%) $(p<0.0001)$. ICM features were more strongly correlated to grade than FCM features. In multivariate analysis, the best discriminating set of features was DNA ploidy and SPF (both by ICM). Conclusions: The adapted fully automated DNA ICM works very well for UCs. Low CV DNA ICM histograms are obtained in a time comparable to FCM. The DNA ICM results have stronger discriminative power than DNA FCM for grade in TaT1 UCs.

Colour figures can be viewed on http://www.esacp.org/acp/2003/25-3/bol.htm.
\end{abstract}

Keywords: Urothelial carcinoma, urinary bladder, grade, flow cytometry, image cytometry

\section{Introduction}

Grading is important in TaT1 urothelial cell carcinomas of the urinary bladder (UCs) as therapy partly depends on grade [11]. However, grade is not well reproducible [17]. This carries the risk of serious over- and under treatment. Therefore the need for objective and reproducible features in UCs is high. Previous stud-

*Address for correspondence: Professor J.P.A. Baak, SIR Hospital, Stavanger, Norway. Tel.: +47 (51) 519366; Fax: +47 (51) 519910; E-mail: baja@sir.no. ies on UCs have found that DNA ploidy is correlated with grade [21]. Flow cytometry (FCM) has mostly been used for ploidy measurements $[9,34]$ but other studies have applied image cytometry (ICM) [22,24]. DNA FCM has the advantage of rapid measurement of a large number of cells. The measurement precision is high, allowing the identification of small deviations in DNA content of a specimen. However, there are several disadvantages of FCM. An aneuploid peak may not be observed if the chromosome complement is within one or two times the standard deviation of the normal ("near diploid/aneuploid") peak. If the num- 
ber of aneuploid cells is small, the excessively larger number of diploid cells in the histogram may obscure low frequency aneuploid tumour cell lines. Furthermore there is lack of visual control, and artefact rejection is not possible on standard inexpensive flow cytometers used in most laboratories. Finally, there is lack of topographical information so that mixtures of different cells (benign, malignant) are measured, relatively large amounts of tissue are needed and measurements are hard to repeat $[19,24]$. Although multiparameter FCM has the capability to considerably reduce the disadvantages of conventional FCM [20] in comparison with image cytometry, the technique is much more expensive and it also requires highly trained and motivated operating staff.

DNA ICM has several advantages over DNA FCM, the most important being the possibility to select epithelial cancer cells for measurement, the sample will still be available for further measurements and relocation of cells of interest is possible afterwards. Historically, a disadvantage of ICM systems was low speed. However, several ultrafast computerised image analysis systems for ploidy measurements have emerged over the past two decades [5] and several studies have shown that DNA ICM gives prognostic results that are superior to classical grading [29, 30]. Moreover, image cytometers can be used for measurements of cell and tissue features other than DNA. The laboratory type image cytometer developed by us also allowed screening for mitoses in tissue sections $[2,16,32]$, measurement of micronuclei [3], quantitation of epithelium in ovarian-, and endometrial carcinomas [25,26], and counting of microvessel density in breast cancer sections [4]. A recent study on breast cancer showed a high measurement accuracy of the DNA image cytometer using the possibility to pre-select tumour cell nuclei only, by applying geometric and densitometric filters [5]. In that study complete concordance between DNA FCM and DNA ICM ploidy classes was $65 \%$ only and the results of ICM seemed superior to those of FCM.

However, evaluation of the thresholds for DNA ploidy and S-phase fraction (SPF) in UCs has not been performed. Thus, in the present study it is analysed whether the pre-set automatic geometric and densitometric thresholds used for automated selection of objects for measurements in breast cancer also hold for UCs and, if necessary, to adapt and test the DNA ICM again. The ICM results will be compared with FCM.
Furthermore, the discriminative power of DNA FCM and ICM features in relation to grade will be evaluated.

\section{Materials and methods}

\subsection{Patients}

Two-hundred-and-sixty-eight consecutive TaT1 UCs were obtained by trans urethral resection (TUR) or by biopsy, fixed in $4 \%$ buffered formaldehyde, dehydrated and embedded in paraffin. Newly Haematoxylin-Eosin (HE) stained $4 \mu \mathrm{m}$ sections were made. The samples used for analysis showed the characteristic TaT1 UC lesion. The worst differentiated area of the HE section was carefully demarcated with a black marker (= measurement area, minimally $2 \times 2 \mathrm{~mm}$ and maximally $5 \times 5 \mathrm{~mm}$ ) and graded according to the WHO classification [18]. Consensus stage and grade was reached at 228/268 (85\%) (44 grade 1, 94 grade 2, 64 grade 3 ).

\subsection{Specimen preparation}

Exactly the same measurement area used for grading was demarcated with a sharp knife on the paraffin embedded tumour tissue for single cell preparation used for FCM and ICM. Fifty $\mu \mathrm{m}$ thick rolls were prepared followed by a $4 \mu \mathrm{m}$ HE stained section to check for the presence of the representative tumour parts ("sandwich technique"). Cell suspensions were prepared according to the Hedley procedure (using Pronase XXVII (Sigma, $0.0005 \mathrm{mg} / \mathrm{ml}$ PBS) treatment for 30 minutes at $37^{\circ} \mathrm{C}$ ) [15]. Care was taken that the cell suspension was homogeneous and part of the cell suspension was stained with DAPI for FCM. For FCM satisfactory cell suspensions could be made for 216/228 UCs (94.7\%). Cytospins were prepared and Feulgen stained according to the consensus of the European Society for Analytical Cellular Pathology (ESACP) $[13,14]$. In short, cytospins were treated with $5 \mathrm{~N} \mathrm{HCl}$ for 30 minutes at a carefully controled air-conditioned room temperature of $20^{\circ} \mathrm{C}$, rinsed in distilled water for 5 minutes, stained with fresh Schiff reagent for 45 minutes and washed in running tap water for 15 minutes. Because DNA FCM measurements were made first in 14 cases there was not enough material left for ICM, thus leaving 202/216 (93.5\%) satisfactory cytospins, and these cases were further considered. 


\subsection{FCM and ICM}

In the current study all samples were analyzed within 3 hours after staining on a commercially available flow cytometer (PARTEC, Munster, Germany). The coefficient of variation $(\mathrm{CV})$ was defined as the ratio of the half-width at $61 \%(2 \times$ standard deviation $)$ of the G0/G1 peak on the abscissa. Trout erythrocytes served as external control cells.

The original, fully automated DNA image cytometer used was developed and evaluated over a period of 15 years in our laboratory [27,28,31,32]. Recently, all basic principles were implemented in a commercially available system (QPATH, Leica, Cambridge, England). The system was carefully evaluated in a European concerted action (CAAC) as described before by Belien [5]. It consists of a Leica LB transmission light microscope with a PRIOR motorized scanning stage and autofocus system. A black-white Kohu Camera was used. The system follows the guidelines of the consensus report of the ESACP [13,14]. Image data as well as numerical data resulting from measurements can be stored in combined databases. The image system settings were used as follows. A voltage stabilizer assured $220 \mathrm{~V} \pm 1 \%$. Before every image analysis session Köhler illumination was applied, and the camera was warmed up for at least 30 minutes to ensure standardized conditions. The images were linearly corrected for shading with a commercially available calibration grid with different increasing optical densities. The corrected grey values thus provided a measure for the local optical density. Segmentation was done fully automatically based on the algorithm described by Vossepoel et al. [33]. Diploid rat liver reference cells were used as external control cells.

\subsection{Filter development for automated DNA ICM}

Densitometric and geometric filters to remove debris, leukocytes, fibrocytes, and aggregates were active during the measurement. At the start of the study, the filters developed for breast cancer [5] were used. We started with a learning set of 45 consecutive cases. It soon appeared that the image processing filters developed for breast cancer performed poorly on the UC specimens and the filters then were adapted as follows. Three thousand random objects per specimen $(135,000$ objects in total) were characterised cytologically by two of us (BD, JB) as epithelial cancer cells, noncancer epithelial cells, inflammatory cells, fibrocytes, cell debris or cluster. This was done interactively by review on the monitor and in the microscope (if necessary). From this database, the different objects were then classified on the basis of the quantitative features (using the different cell types as dependent variable). There was considerable variation in the quantitative features of the same cell type from one specimen to the other and we used the features of all objects of the 45 cases to train the system. This resulted in a classification algorithm which then was tested on an independent set of 20 new cases, which were unambiguously classified as diploid or aneuploid by FCM and also had low CV's of the diploid peaks. The results were already highly satisfactory, and only slight filter adaptations had to be carried out. This pre-final testing algorithm was then applied to 10 additional cases. No further adaptations appeared necessary and the DNA ICM method optimized for UCs was subsequently applied to all cases. The image database of each case was again evaluated, and less than 298 objects per histogram had to be rejected by the operator (average rejection rate 152 objects, range 84-298). Overall measurement time of the adapted ICM was 10.7 minutes (range 5.9-29.8 $\mathrm{min}$ ).

\subsection{Histogram analysis}

In the histograms, the $2 \mathrm{c}$ peak was identified interactively, after which the histogram was scaled up to 10c with a fixed number of 256 bins in order to obtain standardised histograms for all cases. After the measurement the histograms were filed in ASCII format. FCM and ICM DNA histograms were analysed with the MultiCycle AV computer program (Phoenix Flow Systems, San Diego, CA, USA) according to a previously described protocol [6,7]. From the histograms the DNA index (DI) was determined as the ratio of the second G0/G1 to the first G0/G1 peak in the DNA histogram assuming that the first G0/G1 peak to the left belonged to the diploid cells, either from the tumour cells or from other cells present in the specimen. A diploid (2c) tumour showed only the G0/G1 peak in the expected region in the histogram. Diploid tumours were defined as DI $=1.00$. An aneuploid tumour showed an additional peak to the $2 \mathrm{c}$ peak. Near-diploid/aneuploid tumours were defined as $1.00<$ DI $<1.30$. Low aneuploid tumours had a $1.30<$ DI $<1.90$. Tetraploid tumours showed a G0/G1 peak on a double distance of the diploid peak with a $1.90<\mathrm{DI}<2.10$. The $4 \mathrm{c}$ peak should have a height of at least $10 \%$ of the diploid peak to be classified as tetraploid. High aneuploid tumours were de- 
fined as DI $>2.10$. The $\mathrm{CV}$ of the diploid peak was defined as the ratio of the half-width at $61 \%(2 \times$ standard deviation) of the G0/G1 peak on the abscissa.

\subsection{Statistics}

Statistical analysis was performed with SPSS for Windows 10.0 (SPSS, Chicago, USA). The following features were included in the analysis: grade, DNA ploidy of the FCM and ICM, DNA ploidy of the FCM and ICM into five groups (as described above), SPF from FCM and ICM and CV of FCM and ICM. Descriptive analysis was performed and box- and scatter plots were made. The differences of the mean values of the DNA ploidy, DNA ploidy into five groups, SPF and CV for FCM and ICM were analysed for the total group with a Wilcoxon signed rank test. Multivariate analysis was performed to determine the best combination of discriminating features between the grades 1, 2, and 3. A linear discriminant function was derived for the two pairs of neighbouring grades and the percentage of correctly classified cases was assessed.

\section{Results}

Colour figures can be viewed on http://www.esacp. org/acp/2003/25-3/bol.htm

The newly developed filters for ICM resulted in 202 DNA histograms that consisted of at least 2702 and maximally 2916 urothelial tumour cell nuclei (after rejection by the operator). The CV of ICM is considerably lower than that of FCM, and the ICM histograms looked "cleaner". Figure 1 shows the correlation between the DI of the FCM and ICM. There is a reasonably good correlation $(r=0.69, P<0.0001)$, but with exceptions. In total there are 29 (14\%) cases that are aneuploid by FCM and diploid by ICM, and vice-versa. Table 1 shows the distribution of ploidy classes into 5 groups for FCM and ICM. In 160/202 cases $(79.2 \%)$ complete agreement occurred between FCM and ICM ploidy classes. The number of diploid cases is comparable (128 versus 133), but with FCM the number of near diploid/aneuploid cases is higher than with $\operatorname{ICM}(10 / 202=4.4 \%$ vs. $3 / 202=1.3 \%)$, and ICM classifies slightly more cases as low aneuploid $(45 / 202=22.3 \%$ vs. $40 / 202=19.8 \%)$. Twelve (9.4\%) of the 128 diploid cases by FCM are nondiploid by ICM. Discrepancies are most frequent in the near diploid/aneuploid cases by FCM $(9 / 10=90 \%)$. Of these 10 cases, 7 are near diploid/aneuploid by

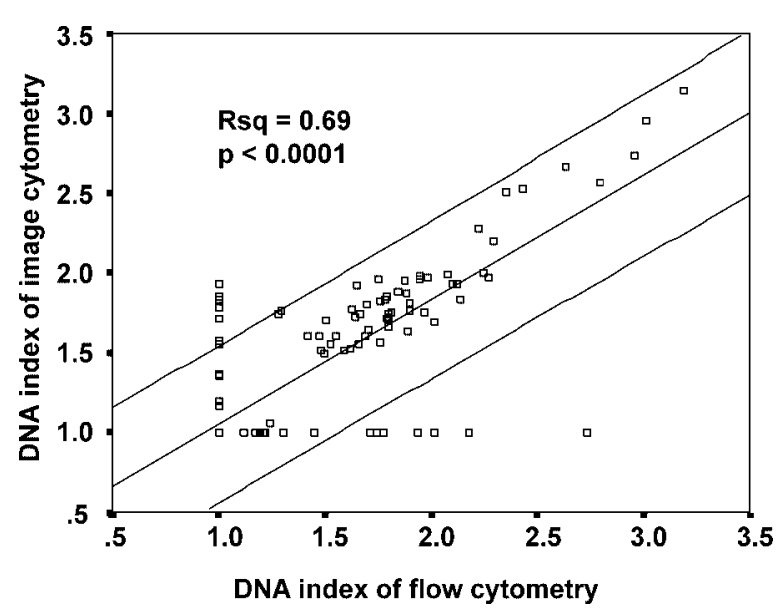

Fig. 1. Urothelial cell carcinomas (UCs). Scatterplot of DNA Indices (DI) obtained by flow cytometry (FCM) and image cytometry (ICM) in 202 cases. Note the correlation but also the variation. The cases with a FCM aneuploid ICM diploid cell line can be caused by clumps not recognized by FCM. The flow-cytometrically diploid ICM aneuploid cases can be caused by the better detection properties of rare near-diploid aneuploid cells.

\begin{tabular}{|c|c|c|c|c|c|c|c|}
\hline \multirow{8}{*}{ FCM } & \multicolumn{7}{|c|}{ ICM } \\
\hline & DNA ploidy & One & Two & Three & Four & Five & Total \\
\hline & One & 116 & 2 & 9 & 1 & 0 & 128 \\
\hline & two & 7 & 1 & 2 & 0 & 0 & 10 \\
\hline & Three & 5 & 0 & 31 & 3 & 1 & 40 \\
\hline & Four & 2 & 0 & 2 & 4 & 0 & 8 \\
\hline & Five & 3 & 0 & 1 & 4 & 8 & 16 \\
\hline & Total & 133 & 3 & 45 & 12 & 9 & 202 \\
\hline
\end{tabular}

Table 1. Urothelial cell carcinomas (UCs). Confusion table for the distribution of ploidy classes into 5 groups for DNA flow cytometry (FCM) and DNA image cytometry (ICM). Diploid tumours $=1$, near-diploid/aneuploid tumours $=2$, low aneuploid tumours $=3$, tetraploid tumours $=4$, and high aneuploid tumours $=5$. Overall agreement is $116+1+31+4+8=160 / 202=79.2 \%$

FCM and diploid by ICM. This is probably due to flow cytometrically diploid objects being clumps that are automatically filtered out by ICM. This explanation is in agreement with Figs 2, 3, and 4, showing the scatter plots of DI and SPF for FCM and ICM. Note the much lower values for SPF cells with ICM.

Grade was strongly correlated to DI, SPF and DNA ploidy into 5 classes both for FCM and ICM. However with FCM there was more overlap and SPF of FCM was only weakly significant. Of the 44 grade 1 cases, 3 were aneuploid by FCM, but none by ICM. Overlap between the grades 2 and 3 cases was slightly more outspoken with FCM than with ICM. Of the 94 


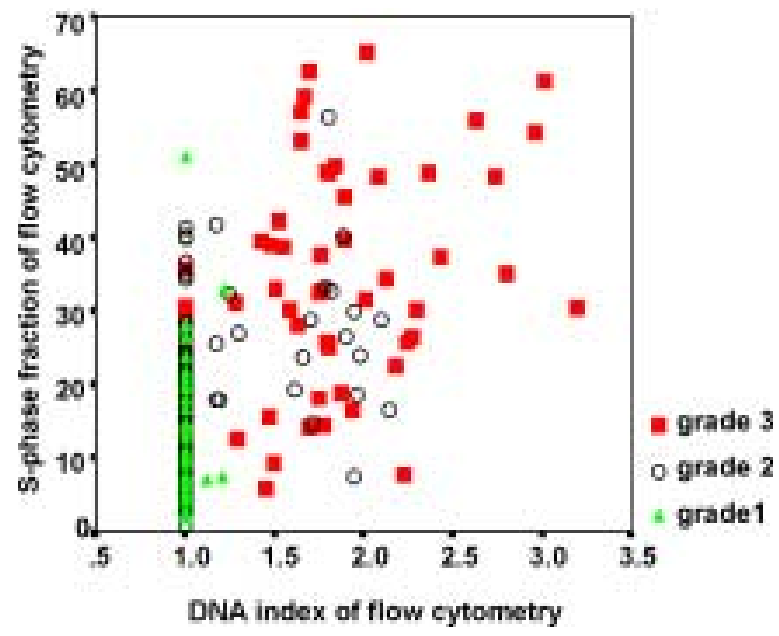

Fig. 2. Urothelial cell carcinomas (UCs). Scatterplot of DNA index (DI) and S phase fraction (SPF) by flow cytometry (FCM) in 202 cases. Note the high values of SPF. Closed triangle; grade 1, open circle, grade 2 ; closed square, grade 3 . This figure can be viewed on http://www.esacp.org/acp/2003/25-3/bol.htm

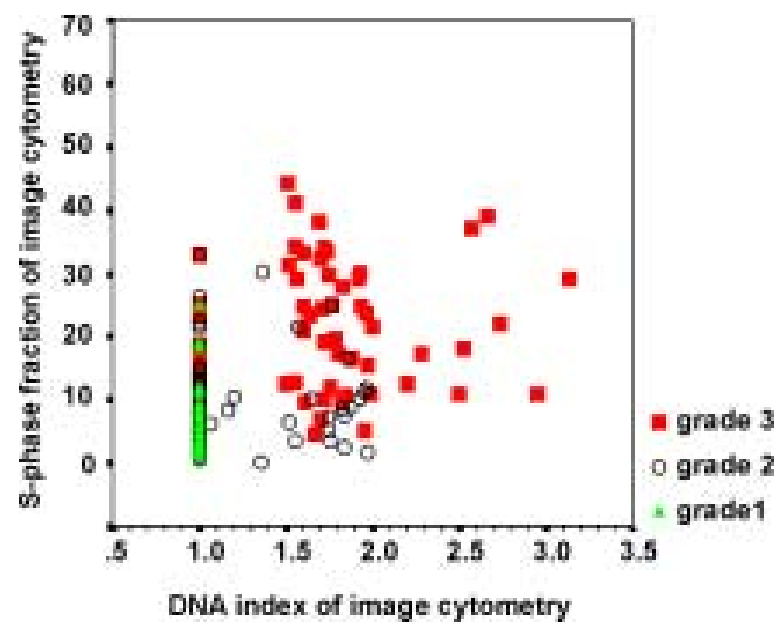

Fig. 3. Urothelial cell carcinomas (UCs). Scatterplot of DNA index (DI) an S phase fraction (SPF) by image cytometry (ICM) in 202 cases. Note the much lower values of SPF compared to SPF by flow cytometry (FCM). Closed triangle; grade 1, open circle, grade 2; closed square, grade 3 . This figure can be viewed on http://www. esacp.org/acp/2003/25-3/bol.htm

grade 2 cases, 72 cases were diploid by FCM and 71 by ICM. Some of the grade 1 cases had very high SPF by FCM, but not with ICM. For FCM it was difficult to find a good combination of features to discriminate between the different grades. In multivariate analysis, ICM features discriminated better than FCM. The best set of discriminating features for grade $1 \mathrm{vs}$. grade 2 was DNA ploidy into 5 classes and SPF $(58.0 \%$ cor-

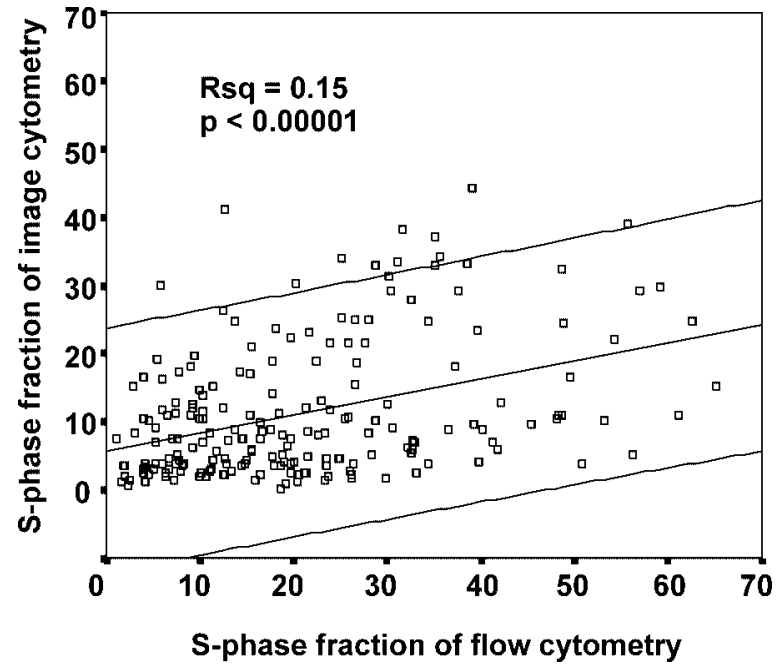

Fig. 4. Urothelial cell carcinomas (UCs). Scatterplot of S phase fraction (SPF) obtained by flow cytometry (FCM) and image cytometry (ICM) in 202 cases.

rectly classified). The best discriminating features for grade 2 vs. grade 3 was DI and SPF of ICM (83\% correctly classified).

\section{Discussion}

The aim of this study was to evaluate how a commercially available automatic DNA image cytometer, developed for breast cancer, performs in UCs, and to analyse in UCs how DNA ploidy and SPF by FCM and ICM correlate with grade. The filters used for breast cancer did not hold for UCs and therefore new ICM filter thresholds had to be developed. These newly developed geometric and densitometric filter thresholds for DNA ploidy and SPF for the automated image cytometry system worked very well for UCs as they gave excellent histograms, were very time effective, and the ICM results correlated better than FCM with grade. Discrimination between grade 1 and grade 2 was not very good which can be explained by the heterogeneity of the grades 2 . This is a well-known phenomenon [8, 10,22]. However, discrimination between grade 2 and grade 3 was satisfactory. It may be necessary to draw an artificial decision line between grade 1 and 2, in order to have an objective criterion for grading support. Indeed, pathologists often feel insecure when they are pushed to distinguish reproducibly between grades 1 and 2. This is understandable as grades form a continuous spectrum of changes and classification shifts are well known in such lesions [1]. Quantitative well re- 
producible techniques as the DNA ICM described can be used to support the decision-making procedure.

DI of the ICM correlated fairly well with DI of the FCM. The correlation coefficient of 0.69 is in concordance with that found in other studies. Some found higher correlation coefficients [12] (often using fresh material), but others [5] found lower correlation coefficients. ICM and FCM detected about equal numbers of aneuploid cases, but many of the FCM specimens contained clumps. When using a DNA ploidy classification into five groups, complete concordance between ICM and FCM was $79.2 \%$. When a more simplified grouping into DNA diploid versus aneuploid was applied (which is clinically probably more useful), the concordance was $85.3 \%$. These results again parallel those of others [5]. Artifacts in the preparation procedures and differences in the interpretation of the DNA histograms may explain the discrepancies in DNA ploidy classification. For instance, DNA aneuploid peaks detected by ICM may be overshadowed by large numbers of non-tumour cells in FCM, and DNA near diploid/aneuploid peaks detected by FCM may be due to clumping which is excluded in ICM. Theoretically, intra tumour heterogeneity may play a role in the analysis of DNA content but this is largely ruled out in the present study where FCM and ICM were done on the same cell sample.

Characteristic primary chromosomal aberrations associated with UCs are the loss of chromosome 5, monosomy 9, deletions in the short arm of chromosome 11, and deletion of a fragment of chromosome 19. Secondary chromosomal anomalies frequently occur on chromosomes 1, 7, 11, 17, and others [23]. This has been compiled into a commercially available multiparameter FISH test (UroVysion), but the cost of one test is fairly high. It remains to be determined whether it performs better diagnostically and prognostically than analysis of whole genome DNA aberrations by automated ICM. Currently, automated ICM seems an accurate, time-efficient, and cost-effective alternative for daily practice.

\section{Acknowledgement}

Supported by grant 01-203 of the Stichting Bevordering Diagnostische Morfometrie (SBDM).

\section{References}

[1] J.P.A. Baak, F.A. Langley, A. Talerman and J.F. Delemarre, Interpathologist and intrapathologist disagreement in ovarian tumor grading and typing, Anal. Quant. Cytol. Histol. 8 (1986), $354-357$.
[2] J.A. Belien, J.P.A. Baak, P.J. van Diest and A.H. van Ginkel, Counting mitoses by image processing in Feulgen stained breast cancer sections: the influence of resolution, Cytometry 28 (1997), 135-140.

[3] J.A. Belien, M.P. Copper, B.J. Braakhuis, G.B. Snow and J.P.A. Baak, Standardization of counting micronuclei: definition of a protocol to measure genotoxic damage in human exfoliated cells, Carcinogenesis 16 (1995), 2395-2400

[4] J.A. Belien, S. Somi, J.S. de Jong, P.J. van Diest and J.P.A. Baak, Fully automated microvessel counting and hot spot selection by image processing of whole tumour sections in invasive breast cancer, J. Clin. Pathol. 52 (1999), 184-192.

[5] J.A.M. Belien, J.P.A. Baak, P.J. van Diest, B.N.L.H.M. Misere, G.A. Meijer and L. Bergers, Prognostic value of image and flow cytometric DNA ploidy assessments in invasive breast cancer, Electr. J. Pathol. (1997), 972-979.

[6] E. Bergers, R. Montironi, P.J. van Diest, E. Prete and J.P.A. Baak, Interlaboratory reproducibility of semi automated cell cycle analysis of flow cytometric DNA-histograms obtained from fresh material of 1,295 breast cancer cases, Hum. Pathol. 27 (1996), 553-560.

[7] E. Bergers, P.J. van Diest and J.P.A. Baak, Reproducibility of semi-automated cell cycle analysis of flow cytometric DNAhistograms of fresh breast cancer material, Analyt. Cell. Pathol. 8 (1995), 1-13.

[8] S. Biesterfeld, K. Reus, E. Bayer-Pietsch, A.M. Mihalcea, A. Bocking, DNA image cytometry in the differential diagnosis of endocervical adenocarcinoma, Cancer 93 (2001), 160-164.

[9] H. Bittard, B. Lamy and C. Billery, Clinical evaluation of cell deoxyribonucleic acid content measured by flowcytometry in bladder cancer, J. Urol. 155 (1996), 1887-1891.

[10] A. Bocking, Prognostic relevance of cytometric methods for tumors of the urinary tract and prostate, Verh. Dtsch. Ges. Pathol. 77 (1993), 161-168.

[11] L. Cheng, R.M. Neumann, A.L. Weaver, J.C. Cheville, B.C. Leibovich, D.M. Ramnani, B.G. Scherer, A. Nehra, H. Zincke and D.G. Bostwick, Grading and staging of bladder carcinoma in transurethral resection specimens. Correlation with 105 matched cystectomy specimens. Am. J. Clin. Pathol. 113 (2000), 275-279.

[12] D.A. Ellison, S.J. Maygarden and D.B. Novotny, Quantitative DNA analysis of fresh solid tumors by flow and image cytometric methods: a comparison using the Roche Pathology Workstation Image Analyzer, Mod. Pathol. 8 (1995), 275-281.

[13] F. Giroud, G. Haroske and A. Reith, A. Bocking, 1997 ESACP consensus report on diagnostic DNA image cytometry. Part II: Specific recommendations for quality assurance. European Society for Analytical Cellular Pathology, Analyt. Cell. Pathol. 17 (1998), 201-208.

[14] G. Haroske, F. Giroud, A. Reith and A. Bocking, 1997 ESACP consensus report on diagnostic DNA image cytometry. Part I: Basic considerations and recommendations for preparation, measurement and interpretation. European Society for Analytical Cellular Pathology, Analyt. Cell. Pathol. 17 (1998), 189200

[15] D.W. Hedley, M.L. Friedlander, I.W. Taylor, C.A. Rugg and E.A. Musgrove, Method for analysis of cellular DNA content of paraffin-embedded pathological material using flow cytometry, J. Histochem. Cytochem. 31 (1983), 1333-1335. 
[16] E.J. Kaman, A.W. Smeulders, P.W. Verbeek, I.T. Young and J.P.A. Baak, Image processing for mitoses in sections of breast cancer: a feasibility study, Cytometry 5 (1984), 244-249.

[17] G. Mikuz, The reliability and reproducibility of the different classifications of bladder cancer, in: Surgical Pathology Update 2001, S. Hauptmann M. Dietel and M. Sorbrinho-Simoes, eds, ABW-Wissenschaftsverlag, Berlin, 2001, pp. 114-115.

[18] F.K. Mostofi, C.J. Davis and I.A. Sesterhenn, WHO Histologic Typing of Urinary Bladder Tumors, Springer, Berlin, 1999.

[19] T.J. O'Leary, Flow cytometry in diagnostic cytology, Diagn. Cytopathol. 18 (1998), 41-46.

[20] M.G. Ormerod, B. Tribukait and W. Giaretti, Consensus report of the task force on standardisation of DNA flow cytometry in clinical pathology. DNA Flow Cytometry Task Force of the European Society for Analytical Cellular Pathology, Analyt. Cell. Pathol. 17 (1998), 103-110.

[21] A. Pich, L. Chiusa, A. Comino and R. Navone, Cell proliferation indices, morphometry and DNA flow cytometry provide objective criteria for distinguishing low and high grade bladder carcinomas, Virchows Arch. 424 (1994), 143-148.

[22] B. Planz, C. Synek, T. Deix, A. Böcking and M. Marberger, Diagnosis of bladder cancer with urinary cytology, immunocytology and DNA-image-cytometry, Analyt. Cell. Pathol. 22 (2001), 103-109.

[23] G. Sauter, T.C. Gasser, H. Moch, J. Richter, F. Jiang, R. Albrecht, H. Novotny, U. Wagner, L. Bubendorf and M.J. Mihatsch, DNA aberrations in urinary bladder cancer detected by flow cytometry and FISH, Urol. Res. 25 (1997), 37-43.

[24] F.R. Schapers, J.J. Ploem-Zaaijer, R.P. Pauwels, A.W. Smeets, P.A. van den Brandt, H.J. Tanke and F.T. Bosman, Image cytometric DNA analysis in transitional cell carcinoma of the bladder, Cancer 72 (1993), 182-189.

[25] N.W. Schipper, J.P.A. Baak and A.W. Smeulders, Automated selection of the most epithelium-rich areas in gynecologic tumor sections, Anal. Quant. Cytol. Histol. 13 (1991), 395-402.
[26] N.W. Schipper, A.W. Smeulders and J.P.A. Baak, Evaluation of automated estimation of epithelial volume and its prognostic value in ovarian tumors, Lab. Invest. 61 (1989), 228-234.

[27] A.W.M. Smeulders and L. Dorst, Manual of Quantitative Pathology in Cancer Diagnosis and Prognosis, J.P.A. Baak, ed., Springer-Verlag, Berlin, 1991, pp. 77-104.

[28] A.W.M. Smeulders and T. ten Cate, Accuracy of optical density measurements of cells, I low resolution, Applied Optics 26(8) (1987), 3249-3257.

[29] J. Sudbo, W. Kildal, A.C. Johannessen, H.S. Koppang, A. Sudbo, H.E. Danielsen, B. Risberg and A. Reith, Gross genomic aberrations in precancers: clinical implications of a long-term follow-up study in oral erythroplakias, J. Clin. Oncol. 20 (2002), 456-462.

[30] J. Sudbo, T. Ried, M. Bryne, W. Kildal, H. Danielsen and A. Reith, Abnormal DNA content predicts the occurrence of carcinomas in non-dysplasticoral white patches, Oral. Oncol. 37 (2001), 558-565.

[31] T. ten Kate, TV microscopical image analysis for accurate DNA quantification in pathology, $\mathrm{PhD}$ thesis, Amsterdam, Free University, 1990.

[32] T.K. ten Kate, J.A. Belien, A.W. Smeulders and J.P.A. Baak, Method for counting mitoses by image processing in Feulgen stained breast cancer sections, Cytometry 14 (1993), 241-250.

[33] A.M. Vossepoel, A.W.M. Smeulders and K. Broek, DIODA: delineation and feature extraction of microscopical objects, Comp. Prog. Biomed. 10 (1979), 231-244.

[34] L.L. Wheeless, J.E. Reeder, M.J. O'Connell, R.D. Robinson, J.M. Cosgriff, Y. Fradet, I.N. Frank and A.T. Cockett, DNA slit-scan flow cytometry of bladder irrigation specimens and the importance of recognizing urothelial cells, Cytometry $\mathbf{1 2}$ (1991), 140-146. 


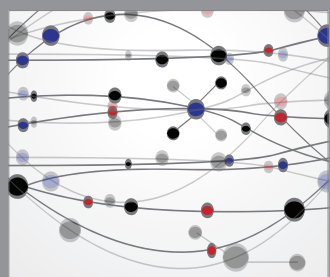

The Scientific World Journal
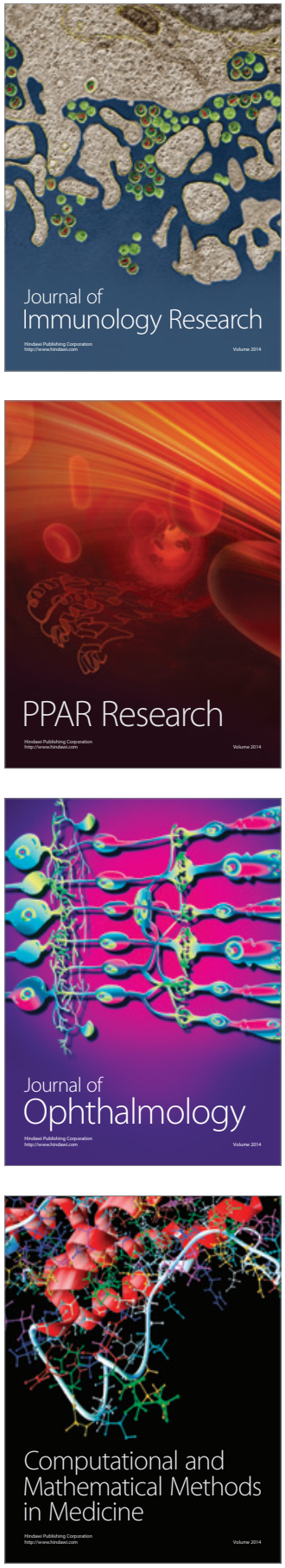

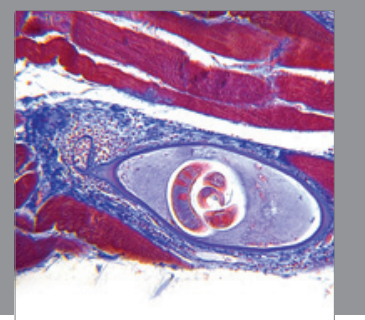

Gastroenterology

Research and Practice
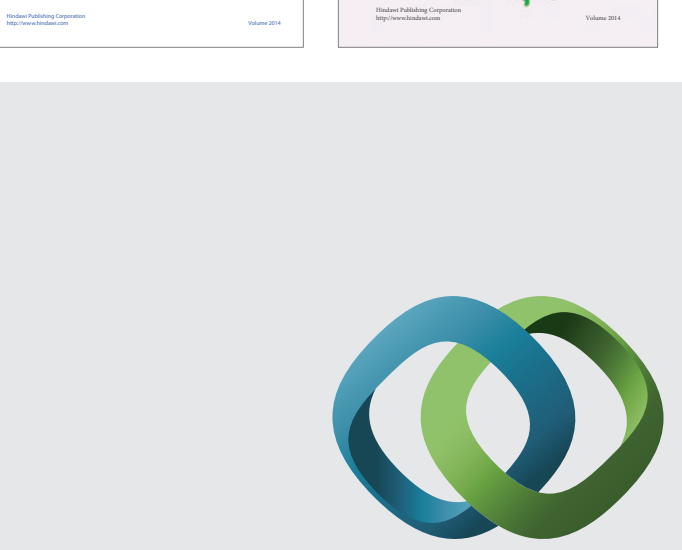

\section{Hindawi}

Submit your manuscripts at

http://www.hindawi.com
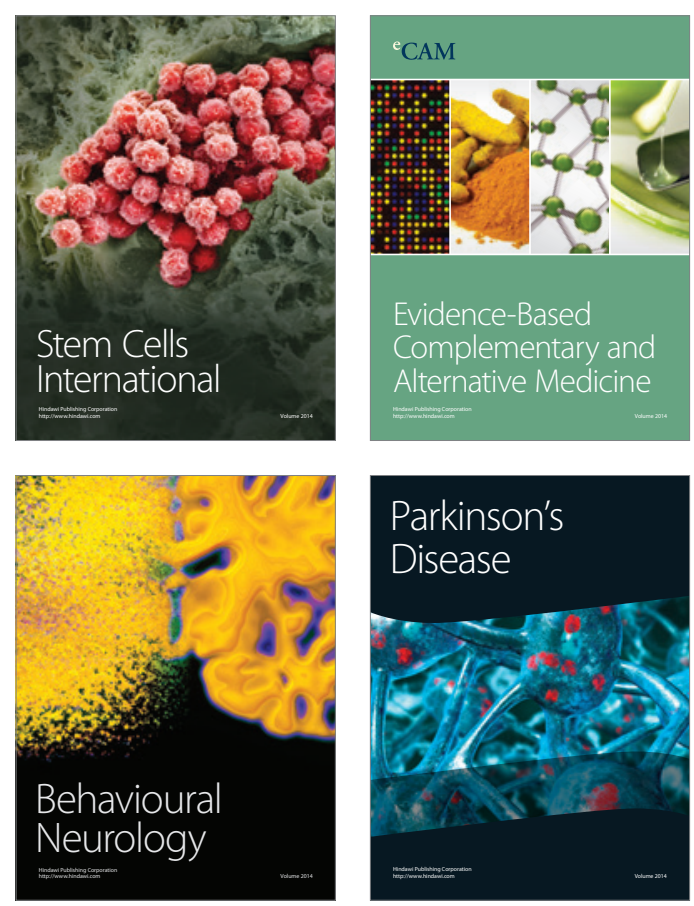

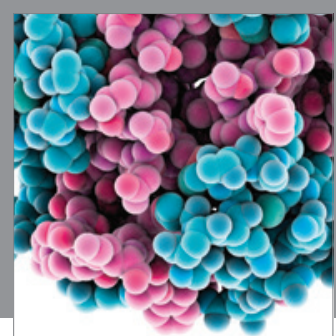

Journal of
Diabetes Research

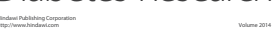

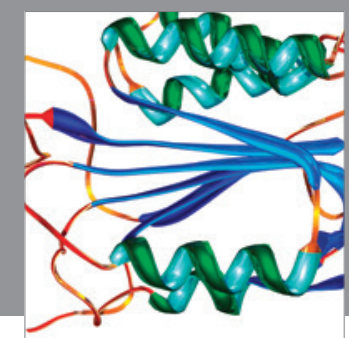

Disease Markers
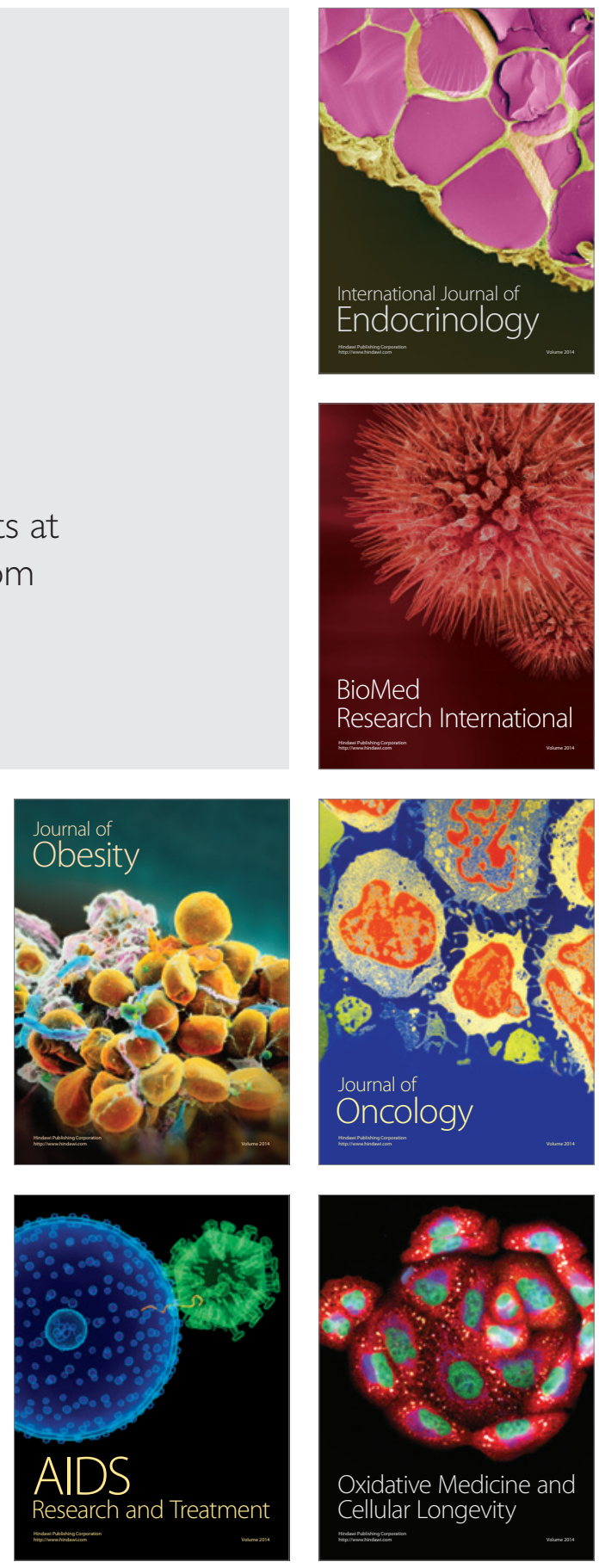